\title{
Predictive Modelling of the COVID - 19 Epidemic in Cameroon with Innovative Models
}

\author{
Jimbo Henri Claver ${ }^{*}$, Ngongo Isidore Séraphin ${ }^{2}$, Fotso Simeon ${ }^{3}$, Waffo Guy ${ }^{4}$, Andjiga Gabriel Nicolas ${ }^{2}$, \\ Etoua Rémy Malgoire ${ }^{5}$ and and Tchantcho Bertrand ${ }^{2}$ \\ ${ }^{1}$ Department of Applied Mathematics and Statistics, RISE, Waseda University, Tokyo, Japan \\ ${ }^{2}$ Department of Mathematics, ENS, University of Yaoundé I, Cameroon \\ ${ }^{3}$ Department of Mathematics, University of Yaoundé 1, Cameroon \\ ${ }^{4}$ Department of Computer Engineering, ENSPY, Cameroon \\ ${ }^{5}$ Department of Mathematics, ENSPY. Yaounde 1, Cameroon \\ Email: jimbo.maths@gmail.com
}

\begin{abstract}
The mathematical model was recently used for COVID-19 in Wuhan, the Chinese city where the disease first broke out. It helped to outline the interventions needed to reduce the number of people at risk of catching the virus. The SEIR models were also used to predict interventions during the 2009 influenza pandemic in the US. In this project we will develop new models that are able to capture more features that the previous ones. Based on the official data modeling, this research work will study the Corona Virus Disease 2019 (COVID-19) propagation dynamics in Cameroon. The error between the model and the official data curve will be estimated. At the same time, it will realize the forward prediction of the epidemic situation, and the relevant analysis will help Cameroon to make important decisions on the epidemic evolution over time.
\end{abstract}

Keywords: Covid 19 modelling, SIR, SSIR, SEIR, SSEIR, model loss, model selection,reproduction number, stability analysis.

\section{Introduction}

Modelling disease propagation is of key importance in epidemiology. In Cameroon, 8681 confirmed cases and 208 deaths of people who tested positive for Covid 19 were registered as of 13 June 2020. Predictive mathematical models for epidemics [15-18] are fundamental to understand the course of the epidemic and to plan effective control strategies. The commonly used model is the SIR model for human-to-human transmission, which describes the flow of individuals through three mutually exclusive stages of infection: susceptible, infected and recovered $[18,19]$. It is generally agreed that more complex models can accurately capture the dynamic spread of specific epidemics. For the COVID-19 pandemic, several models have been developed. [19] extended a SEIR (susceptible, exposed, infected, recovered) model considering risk perception and the cumulative number of cases,[20] proposed a discrete-time SIR model including dead individuals, [21] developed a control-oriented SIR model that stresses the effects of delays and compares the outcomes of different containment policies [22] and [1,2] used transmission dynamics to estimate the clinical severity of COVID-19. Stochastic transmission models have also been considered [24-30]. However stochastic modelling of the state variable have not yet been developed. Here, we propose new approaches to epidemiological model for the COVID-19 epidemic using data from Cameroon and extend the classical SIR model. Ending the global COVID-19/SARS-CoV-2 pandemic requires implementation of multiple population-wide strategies, including social distancing, testing and contact tracing. (see [15-16] for example). These models will predict the course of the epidemic and help to plan an effective control strategy. The stochastic SIR considers at most three stages of infection: susceptible (S), infected (I), recovered (R) collectively termed as SIR with some noise adjustment terms whereas The stochastic SEIR model considers at most four stages of infection: susceptible (S), exposed (E), infected (I), recovered (R) collectively termed as SEIR with some noise adjustments. Our innovative models discriminate between infected individuals depending on whether they have been diagnosed and on the severity of their symptoms implemented in the stochastic part of the models. The distinction between susceptible and infected individuals is important because the former are typically isolated and hence 
less likely to spread the infection[2-4]. We compare simulation results with real data on the COVID-19 epidemic in Cameroon, and we model possible scenarios of implementation of countermeasures. We should mention that there are some interesting mathematical models in the litterature that try to describe the dynamic of the evolution of the covid-19. Three main models can be used to validate the outbreak of the disease. Those models are the SIR, SIRD and SEIR. This is because the Covid-10 is caused by a new virus and generating a world wide emergency situation. The main goal of our paper is to develop new mathematical models well adapted to Covid-19 taking into account the specific caracteristics of the disease [6-10]. Such model should be able to consider different scenarios such as the number of infected people and the number of deaths in the country. They need to be complex enough to capture the most important effects but also simple enough to allow a good identification of the parameters using the data from the government (MINSANTE) and WHO on this pandemic. We propose both deterministic and stochastic models because both models have some advantages such as computational cost, parameter calibration, the use of the theory of differential equations for suitable analysis and the interpretation of the model. Also some authors believe that deterministic models should be the first tool to be used when modelling a new problem with limited data but other authors believe that stochastic models might have some limitations for novel analysis when it is difficult to find the density function or the probability distribution of the model and difficult to analyse as its requires more data for the calibration. Our paper is organized as follows: In section two, we present the old and new mathematical models; in section three we develop the algorithm for optimizing the parameters of the models; in section four we discuss our results; in section five we present the limitations of our results ; we end this work in section six with a conclusion and recommendations

\section{Mathematical Models}

\subsection{Deterministic SIR MODEL}

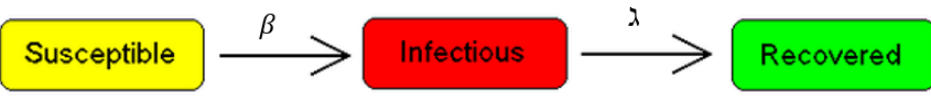

Figure 1. Deterministic SIR

The SIR dynamic system consists of three ordinary differential equations, describing the evolution of the population in each stage over time

$$
\left\{\begin{array}{l}
\frac{d S(t)}{d t}=-\beta \frac{I(t)}{N} S(t) \\
\frac{d I(t)}{d t}=\beta \frac{I(t)}{N} S(t)-\lambda I(t) \\
\frac{d R(t)}{d t}=\lambda I(t)
\end{array}\right.
$$

where $\mathbf{S}(\mathbf{t})$ is the number of susceptible people, $\mathbf{I}(\mathbf{t})$ is the number of infected people and $\mathbf{R}(\mathbf{t})$ is the number of recovered people.

We should notice that $S_{0}=S(0)$.

This system is non-linear, however it is possible to derive its analytic solution in implicit form. Other numerical tools include Monte Carlo methods, such as the Gillespie algorithm.Firstly note that from:

$$
\frac{d S}{d t}+\frac{d I}{d t}+\frac{d R}{d t}=0
$$

It follows that: $S(t)+I(t)+R(t)=$ constant $=N$ 
Reproductive Ratio $R_{0}=\frac{\beta}{\lambda}$ Let $R_{0}$ denote the reproductive ratio. The reproductive ratio is the fundamental parameter governing disease dynamics in our model. Thus we have the following results.

1. $R_{0}<1$ physically interpreted to mean that each person gets the disease will infect less than one person before recovering or dying, so the disease will peter out and eradication is $\frac{d I}{d t}<0$

2. $R_{0}>1$ physically interpreted to mean that each person who gets the disease will infect more than one person, so the epidemic will spread in the host population $\frac{d I}{d t}>0$

We examine the dynamics of the Covid 19 under high transmission rate and high recovery rate. We examine the dynamics of the Covid 19 under low transmission rate with high recovery rate. We have simulated both the existing model as well as our modified model. We have selected some initial values for the numerical experiments and obtained the results.

Theorem 1 The disease-free equilibrium of the model is locally asymptotically stable if $R_{0}<1$, and unstable if $R_{0}>1$. We mention that the basic reproduction number, given in the model equation, can also be derived by the next generation matrix analysis. To study the global asymptotic stability of the DFE, one common approach is to construct an appropriate Lyapunov function. We have found, however, that it is simpler to apply the following result introduced by [17]

Lemma 1 Consider a model system written in the form

$$
\left\{\begin{array}{l}
\frac{d X_{1}}{d t}=F\left(X_{1}, X_{2}\right) \\
\frac{d X_{2}}{d t}=G\left(X_{1}, X_{2}\right) \\
G\left(X_{1}, 0\right)=0
\end{array}\right.
$$

where $X_{1} \in \mathbb{R}$ m denotes (its components) the number of uninfected individuals and $X_{2} \in \mathbb{R}$ denotes (its components) the number of infected individuals including latent, infectious, etc; $X_{0}=\left(X_{1}, 0\right)$ denotes the disease-free equilibrium of the system. Also assume the conditions $\left(H_{1}\right)$ and $\left(H_{2}\right)$ below:

$$
\begin{gathered}
\left(H_{1}\right): \text { for } \frac{d X_{1}}{d t}=F\left(X_{1}, 0\right), X_{1} \text { is globally asymptotically stable } \\
(H 2): G\left(X_{1}, X_{2}\right)=A X_{2}-G\left(X_{1}, X_{2}\right) \\
G\left(X_{1}, X_{2}\right) \geq 0 \text { for }\left(X_{1}, X_{2}\right) \in \Omega
\end{gathered}
$$

where the Jacobian $A=\partial G X_{1} \partial X_{2}\left(X_{1}, 0\right)$ is an $M-$ matrix (the off diagonal elements of A are nonnegative) and $\Omega$ is the region where the model makes biological sense. Then the $D F E X_{0}=\left(X_{1} 1,0\right)$ is globally asymptotically stable provided that $R_{0}<1$.

Theorem 2 The disease-free equilibrium of the model is globally asymptotic stable if $R_{0}<1$.

Proof We only need to show that the conditions $\left(H_{1}\right)$ and $\left(H_{2}\right)$ hold when $R_{0}<1$. In our system of equations, $X_{1}=(S, R), X_{2}=(I, 0)$, and $X_{1}=(N, 0)$. We note that the system $d X_{1} d t=F\left(X_{1}, 0\right)$ is linear and its solution can be easily found as $R(t)=R(0) \exp (-b t)$ and $S(t)=N-N-S(0) \exp (-b t)$ Clearly, $R(t) \rightarrow 0$ and $S(t) \rightarrow N$ as $t \rightarrow \infty$, regardless of the values of $R(0)$ and $S(0)$. Thus $X_{1} 1=(N, 0)$ is globally asymptotically stable.

Theorem 3 In the disease-free-equilibrium(DFE) of the model (N,0,0) and Equilibrium (EE) one can show that, independently from biologically meaningful initial conditions.

$$
(S(0), E(0), I(0), R(0)) \in\left\{(S, E, I, R) \in[0, N]^{4}: S \geq 0, E \geq 0, I \geq 0, R \geq 0, S+E+I+R=N\right\}
$$

it holds that

$$
\begin{gathered}
R_{0} \leq 1 \Longrightarrow \lim _{t \rightarrow+\infty}(S(t), E(t), I(t), R(t))=D F E=(N, 0,0,0), \\
R_{0}>1, I(0)>0 \Longrightarrow \lim _{t \rightarrow+\infty}(S(t), E(t), I(t), R(t))=E E
\end{gathered}
$$

Proof The detailed proofs can be found in [12-22]. 
Deriving the Exact analytical solutions of the SIR model In 2005,[24] derived an exact analytical solution to the SIR model. In the case without vital dynamics setup, for $S(u)=S(t), R(u)=R(t)$

$$
\left\{\begin{array}{l}
S(u)=S(0) u \\
I(u)=N-R(u)-S(u) \\
R(u)=R(0)-\rho \ln u
\end{array}\right.
$$

for $t=\frac{N}{\beta} \int_{u}^{1} \frac{d u *}{u * I(u *)}, \rho=\frac{\gamma N}{\beta}$, with initial conditions

$$
(S(1), I(1), R(1))=(S(0), N-R(0)-S(0), R(0)), \quad u_{T}<u<1,
$$

By dividing the first differential equation by the third, separating the variables and integrating we get:

$$
S(t)=S(0) e^{-R_{0} \frac{R(t)-R(0)}{N}}
$$

An equivalent analytcal solution found by [16] yields

$$
\left\{\begin{array}{l}
S(t)=S(0) e^{-\xi(t)} \\
I(t)=N-S(t)-R(t) \\
R(t)=R(0)+\rho \xi(t) \\
\xi(t)=\frac{\beta}{N} \int_{0}^{t} I(t *) d t *
\end{array}\right.
$$

Here $\xi(t)$ can be interpreted as the expected number of transmissions an individual has received by time $t$. The two solutions are related by $e^{-\xi(t)=u}$. Effectively the same result can be found in the original work by [18]. These solutions may be easily understood by noting that all of the terms on the right-hand sides of the original differential equations are proportional to $t$.

Results The output of deterministic SIR with $S_{0}=10000,20000,40000$ and 100000 is presented in Figure 2, Figure 3, Figure 4, Figure 5 respectively: 


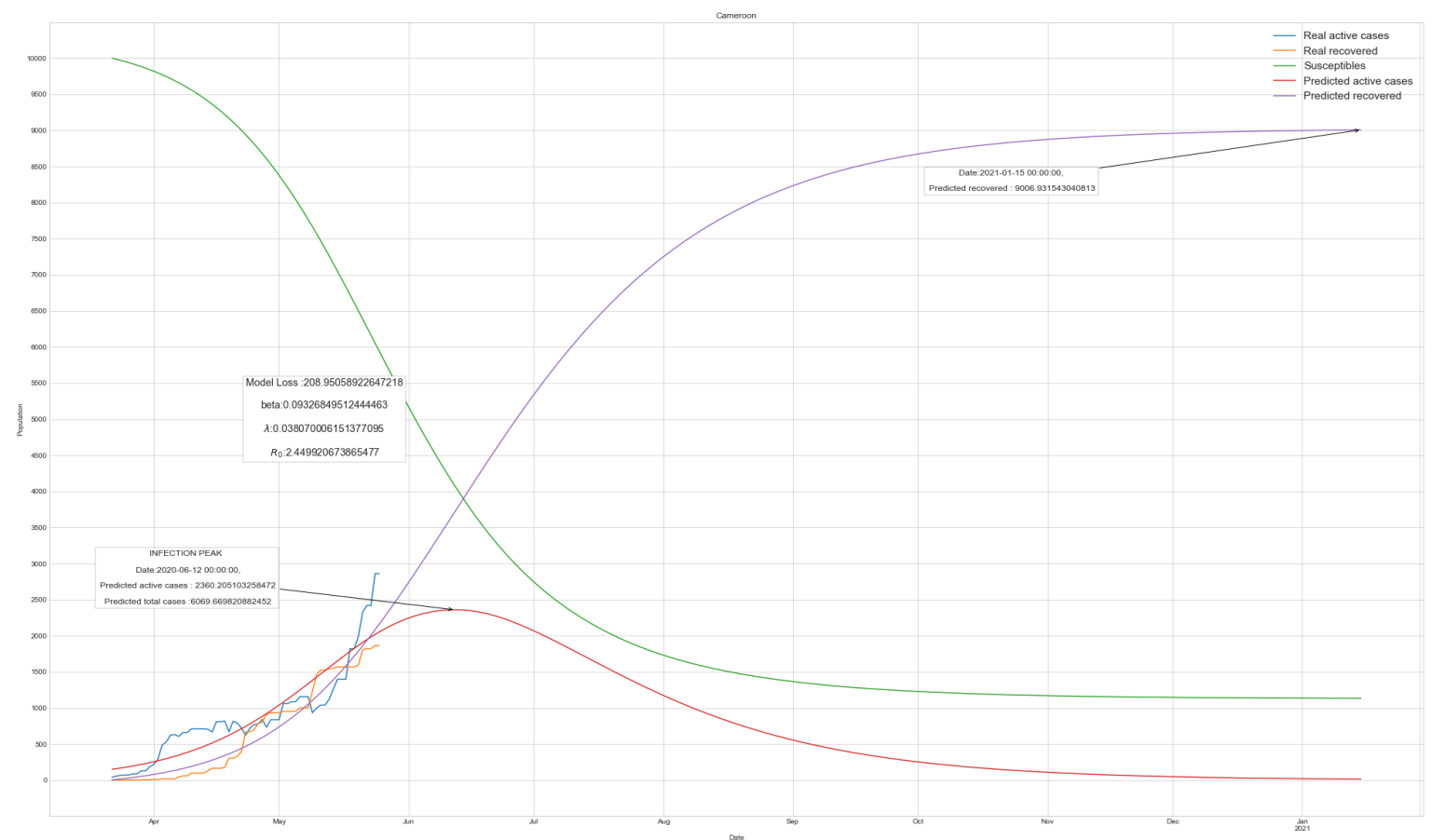

Figure 2. DETERMINISTIC SIR $S_{0}=10000$

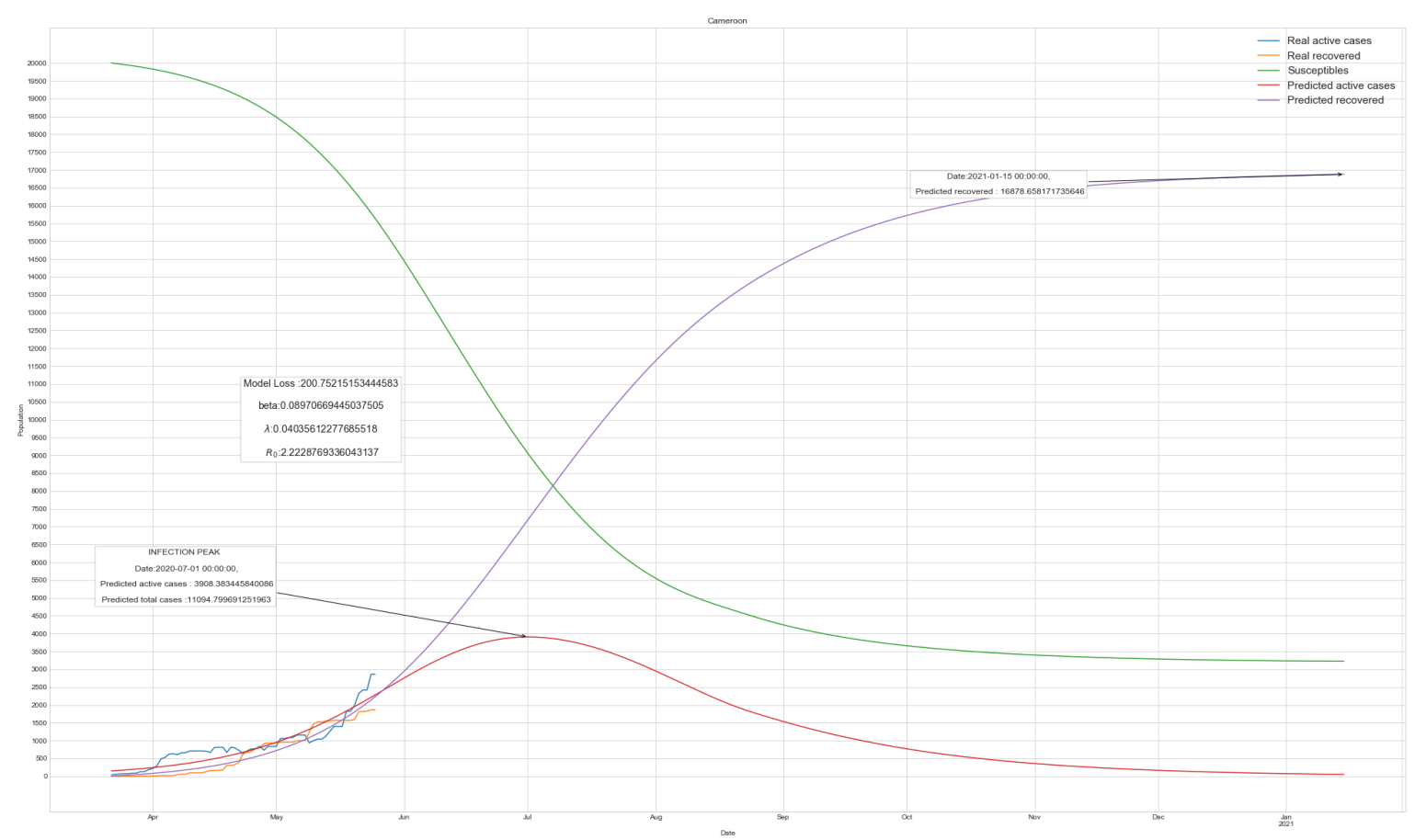

Figure 3. DETERMINISTIC SIR $S_{0}=20000$ 


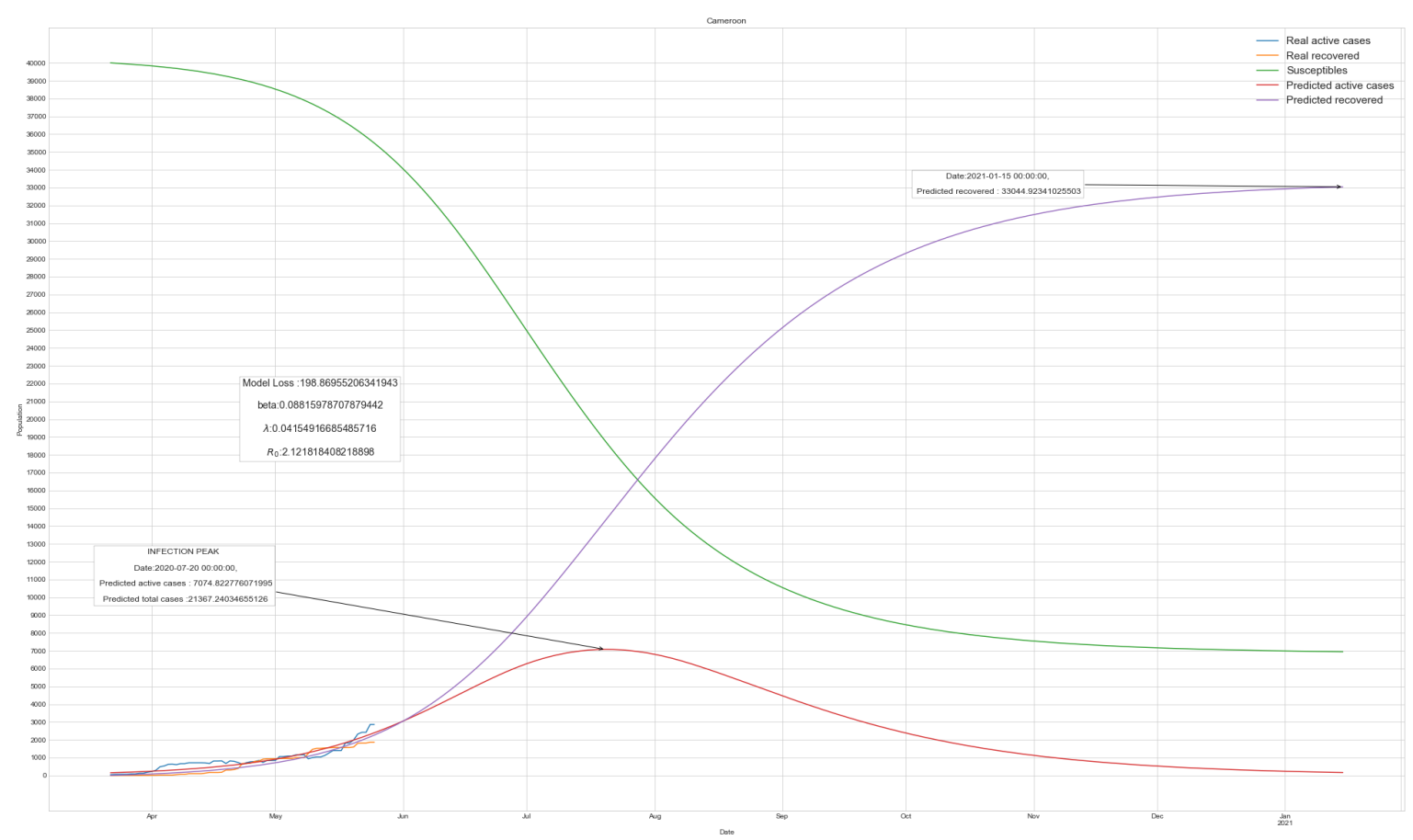

Figure 4. DETERMINISTIC SIR $S_{0}=40000$

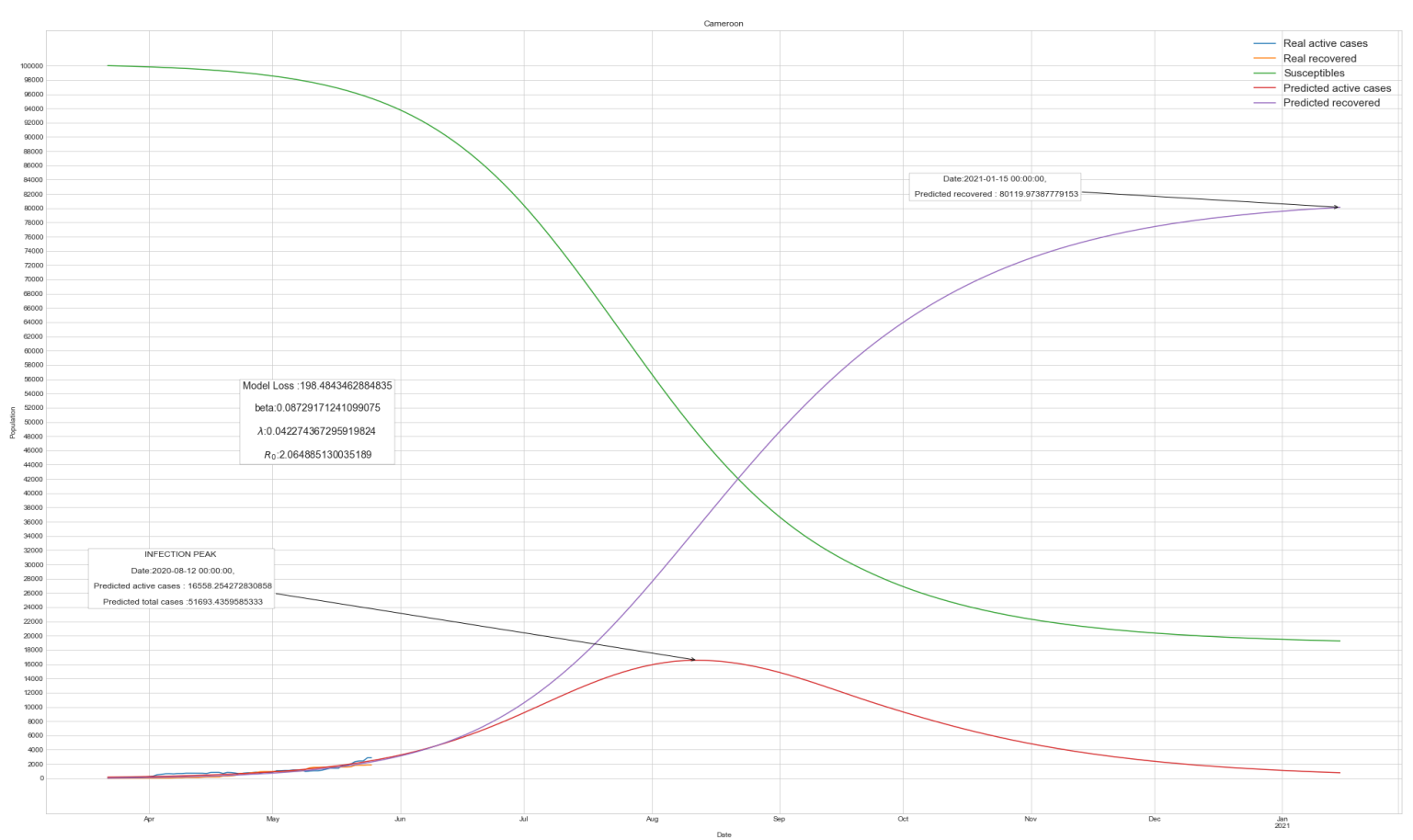

Figure 5. DETERMINISTIC SIR $S_{0}=100000$ 
The parameters of the model defined as $\beta$ and $\lambda$ denote respectively the transmission rate (the probability of disease transmission in a single contact multiplied by the average number of contacts per person) and $\lambda$ is the recovered rate (the probability of recovered individuals multiplied by the average number of contacts per person). Typically, $\beta$ is larger than $\lambda$ in the case of Covid 19 assuming that people tend to avoid contacts with subjects showing symptoms, even though diagnosis has not been made yet). The above parameters can be modified by social-distancing measures, closing schools, remote working, lockdown and so on.

A summary of the main findings, limitations and implications of the model for policymakers is shown in Table 1

Table 1. DETERMINISTIC SIR SUMMARY

\begin{tabular}{llllll}
\hline$S_{0}$ & Infection peak [Total cases] & Peak date & Model loss & $R_{0}$ & Parameters $(\beta, \gamma)$ \\
\hline 10000 & $2360[6069]$ & $12 / 06 / 2020$ & 208.95 & 2.44 & $(0.093,0.038)$ \\
20000 & $3908[11094]$ & $01 / 07 / 2020$ & 200.75 & 2.22 & $(0.089,0.040)$ \\
30000 & $5488[16177]$ & $12 / 07 / 2020$ & 199.32 & 2.154 & $(0.088,0.041)$ \\
40000 & $7074[21367]$ & $20 / 07 / 2020$ & 198.86 & 2.121 & $(0.088,0.004)$ \\
100000 & $15182.82[47457]$ & $10 / 08 / 2020$ & 136.134 & 2.15 & $(0.091,0.042)$ \\
\hline
\end{tabular}

$-S_{0}=S(0)$ is the initial value of the state variable $\mathrm{S}(\mathrm{t})$ (Susceptible)

- Infection peak is the maximum value of the state variable $\mathrm{I}(\mathrm{t})$ representing the exact number of infected person at a given time

- Total cases is the sum of all the infection cases which appeared since the epidemic beginning

- Peak is the date on which Infected peak happens

\subsection{Stochastic SIR Model}

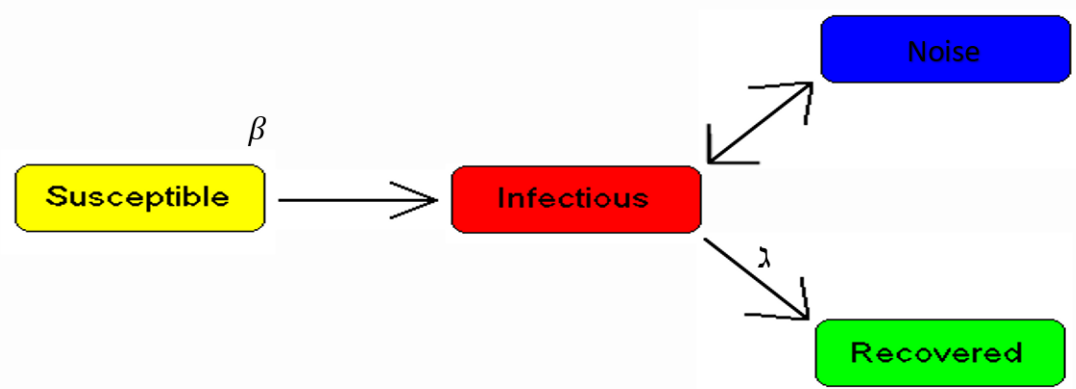

Figure 6. Stochastic SIR

The stochastic SIR model denoted SSIR is an extension of the usual SIR model by adding the noise component in the equation. The noise component stands for all minor impredictible events that might intervent in the dynamic over time. The model is given by:

$$
\left\{\begin{array}{l}
\frac{d S(t)}{d t}=-\beta \frac{I(t)}{N} S(t)+B_{1}(t) \\
\frac{d I(t)}{d t}=\beta \frac{I(t)}{N} S(t)+\lambda I(t)+B_{2}(t) \\
\frac{d R(t)}{d t}=\lambda I(t)+B_{3}(t)
\end{array}\right.
$$

with $\left\{B_{1} \sim \mathcal{N}\left(0, \sigma_{1}\right), B_{2} \sim \mathcal{N}\left(0, \sigma_{2}\right), B_{3} \sim \mathcal{N}\left(0, \sigma_{3}\right)\right\}$. The output of stochastic SIR with $S_{0}=10000$, 20000, 40000 and 100000 is presented in Figure 7, Figure 8, Figure 9 , Figure 10 respectively. 


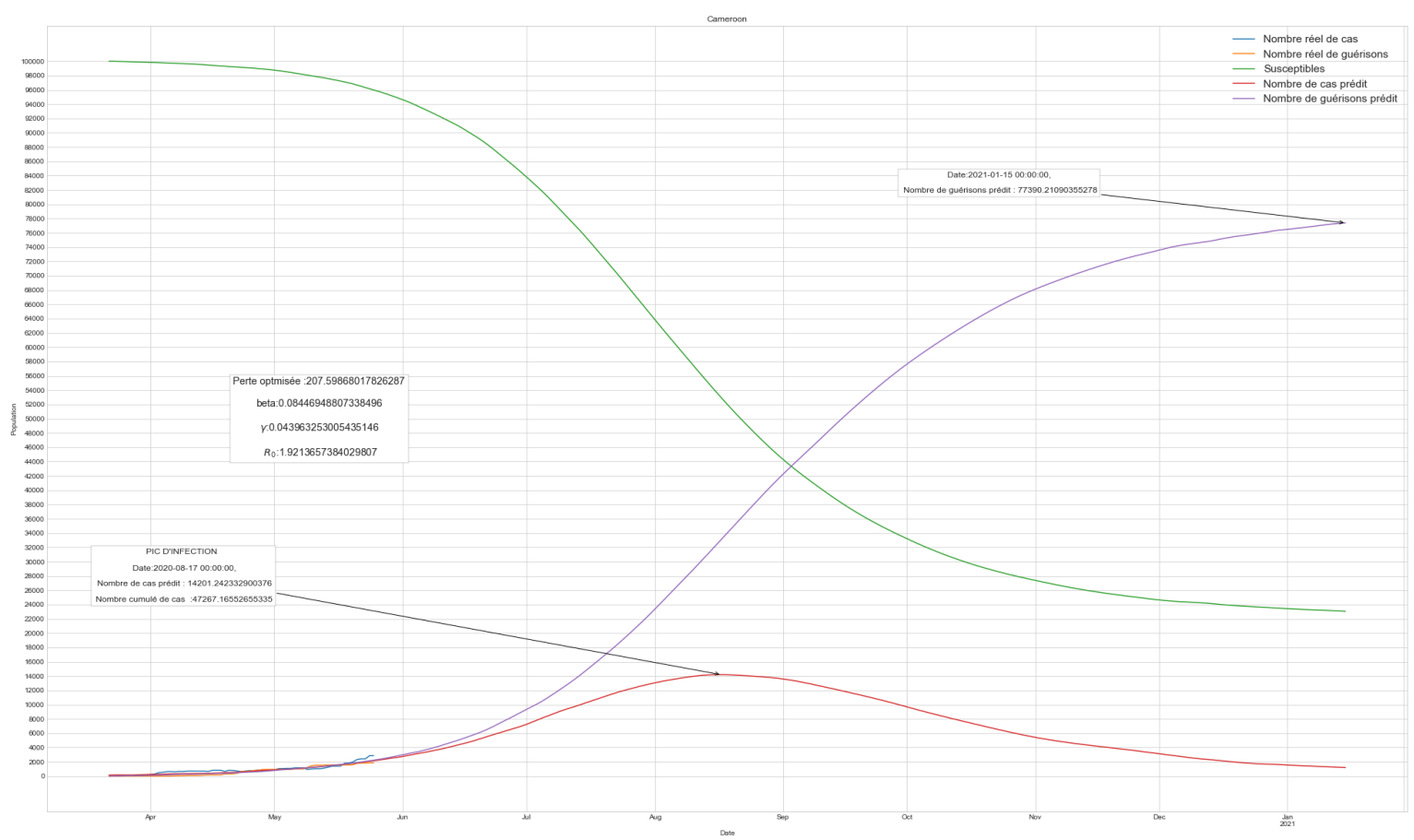

Figure 7. STOCHASTIC SIR $S_{0}=100000$

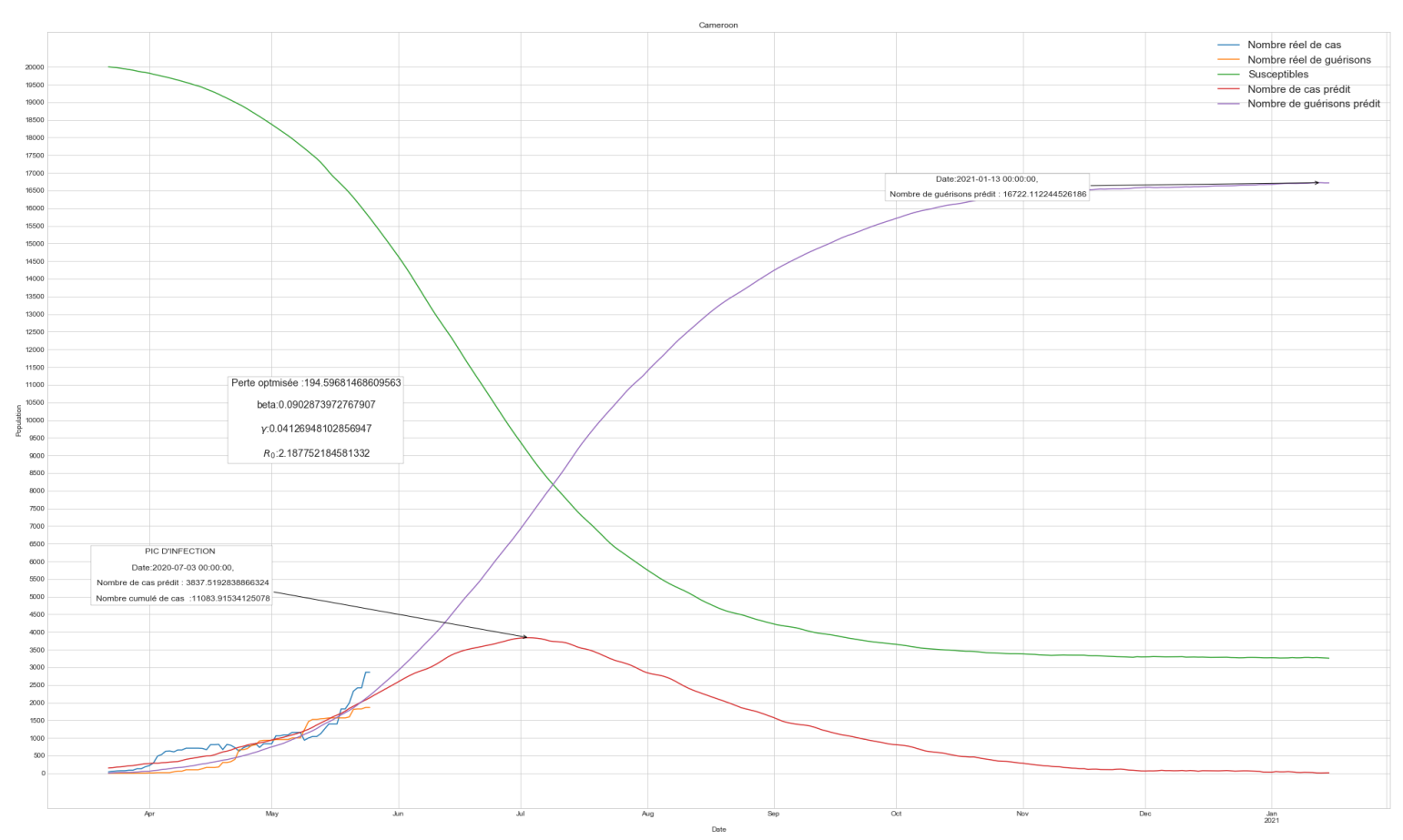

Figure 8. STOCHASTIC SIR $S_{0}=20000$ 


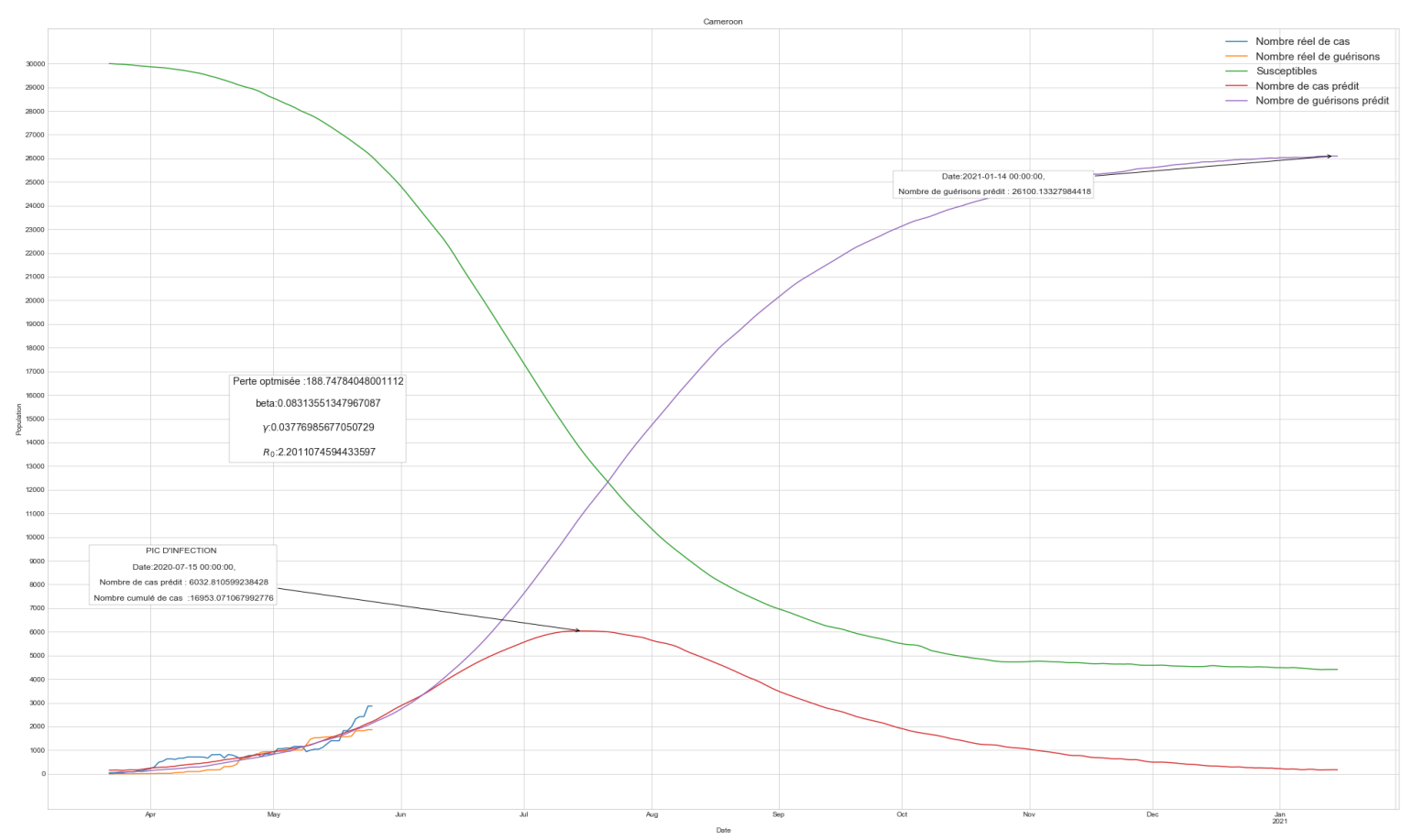

Figure 9. STOCHASTIC SIR $S_{0}=30000$

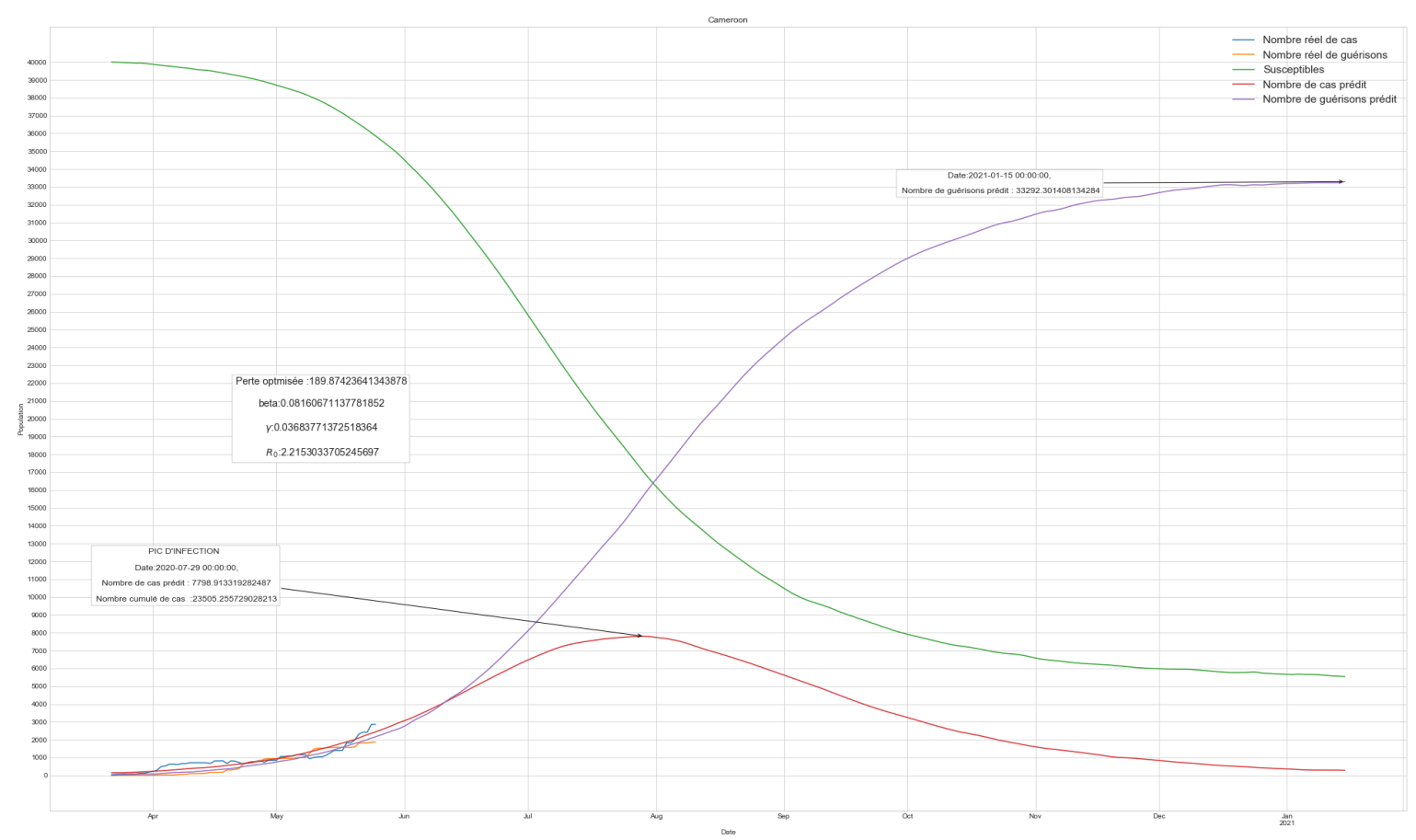

Figure 10. STOCHASTIC SIR $S_{0}=40000$ 
A summary of the main findings, limitations and implications of the model with the date from Cameroon is shown in Table 2

Table 2. STOCHASTIC SIR SUMMARY

\begin{tabular}{|c|c|c|c|c|c|c|}
\hline$S_{0}$ & Noise & $\begin{array}{l}\text { Infection peak } \\
\text { [Total cases] }\end{array}$ & Peak date & $\begin{array}{l}\text { Model } \\
\text { loss }\end{array}$ & $R_{0}$ & $\begin{array}{l}\text { Parameters } \\
(\beta, \lambda)\end{array}$ \\
\hline 10000 & $\begin{array}{l}\mathrm{N}(0,5), \mathrm{N}(0,10) \\
\mathrm{N}(0,10)\end{array}$ & 2364 [6136 ] & 08/06/2020 & 214.69 & 2.326 & $(0.097,0.042)$ \\
\hline 20000 & $\begin{array}{l}\mathrm{N}(0,5), \mathrm{N}(0,10) \\
\mathrm{N}(0,5)\end{array}$ & 3837 [11083] & 03/07/2020 & 194.60 & 2.18 & $(0.090,0.041)$ \\
\hline 30000 & $\begin{array}{l}\mathrm{N}(0,10), \mathrm{N}(0,10), \\
\mathrm{N}(0,10)\end{array}$ & 4684 [15187] & $07 / 07 / 2020$ & 226.92 & 1.95 & $(0.10,0.052)$ \\
\hline 40000 & $\begin{array}{l}\mathrm{N}(0,10), \mathrm{N}(0,5) \\
\mathrm{N}(0,10)\end{array}$ & 7798 [ 21367] & $29 / 07 / 2020$ & 189.874 & 2.21 & $(0.081,0.0036)$ \\
\hline 100000 & $\begin{array}{l}\mathrm{N}(0,10), \mathrm{N}(0,10), \\
\mathrm{N}(0,10)\end{array}$ & $14201[47267]$ & $17 / 08 / 2020$ & 207.5986 & 1.92 & $(0.0814,0.0044)$ \\
\hline
\end{tabular}

$-S_{0}$ is the initial value of the state variable $\mathrm{S}$ (Susceptibles)

- Noise is the description of the noise distribution added to the model. $N(0,10)$ refers to the Gaussian law with

- Infection peak is the maximum value of the state variable I representing the exact number of infected person at a given time.

- Total cases is the sum of all the infection cases which appeared since the epidemic beginning

- Peak is the date on which Infected peak happens

\subsection{Deterministic SEIR Model}

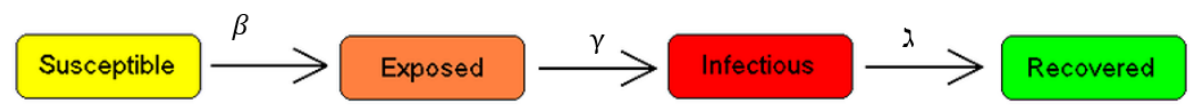

Figure 11. Deterministic SEIR

The deterministic SEIR adds the evolved (E) state variable into the usual SIR model. The equation is as follows:

$$
\left\{\begin{array}{l}
\frac{d S(t)}{d t}=-\beta \frac{I(t)}{N} S(t) \\
\frac{d E(t)}{d t}=\beta \frac{I(t)}{N} S(t)-\gamma E(t) \\
\frac{d I(t)}{d t}=\gamma E(t)-\lambda I(t) \\
\frac{d R(t)}{d t}=\lambda I(t)
\end{array}\right.
$$

The output of deterministic SEIR with $S_{0}=10000,20000,40000$ and 100000 is presented in Figure 12, Figure 13, Figure 14 and Figure 15. 


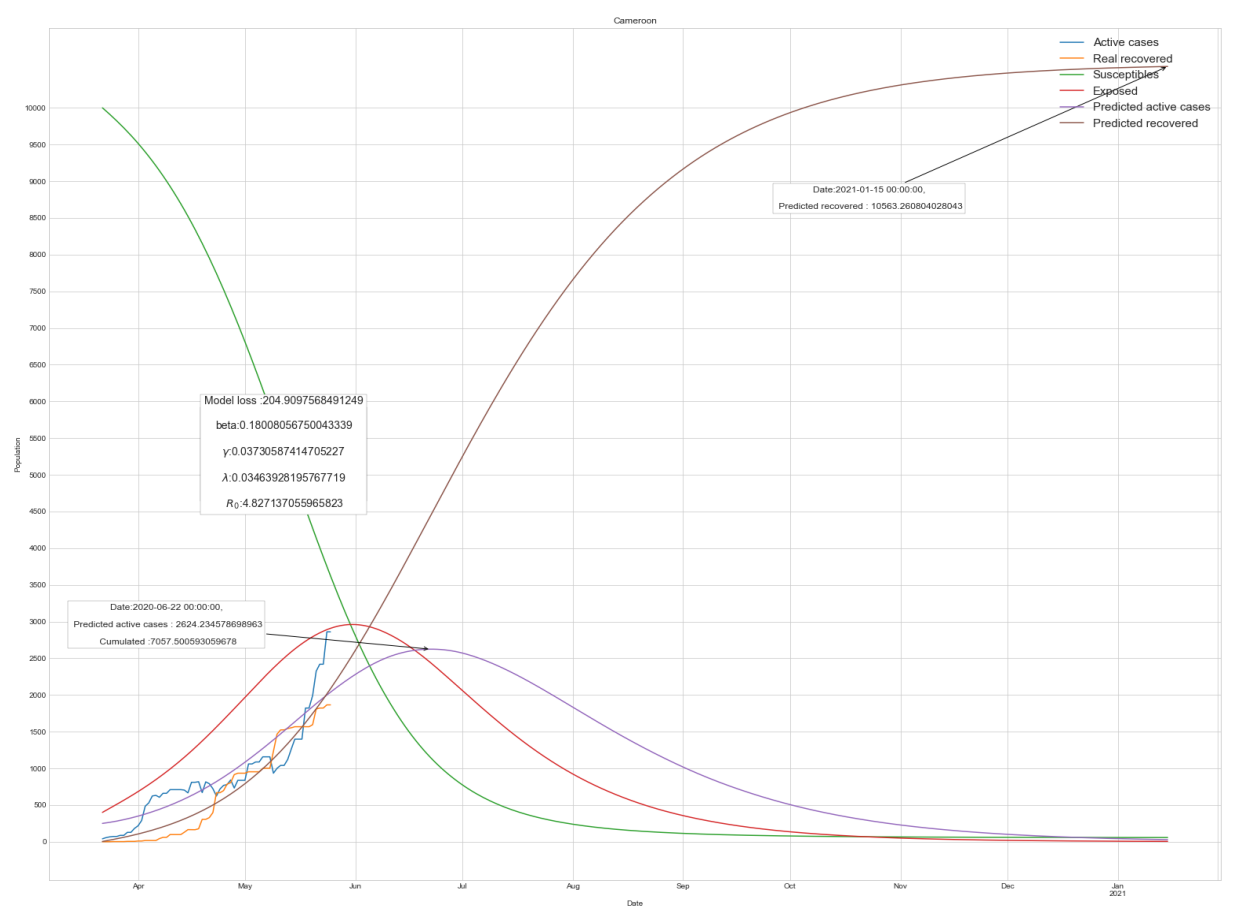

Figure 12. DETERMINISTIC SEIR $S_{0}=10000$

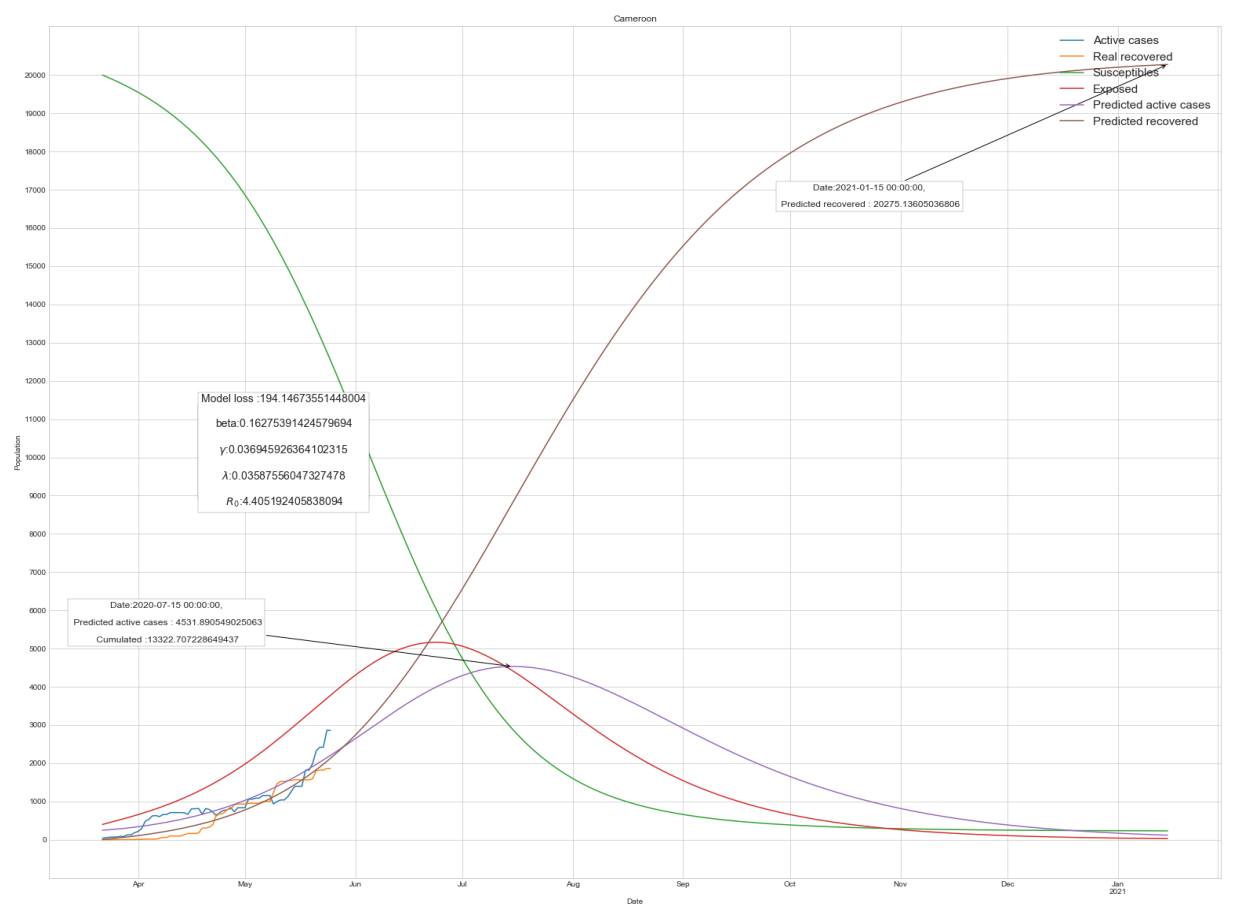

Figure 13. DETERMINISTIC SEIR $S_{0}=20000$ 


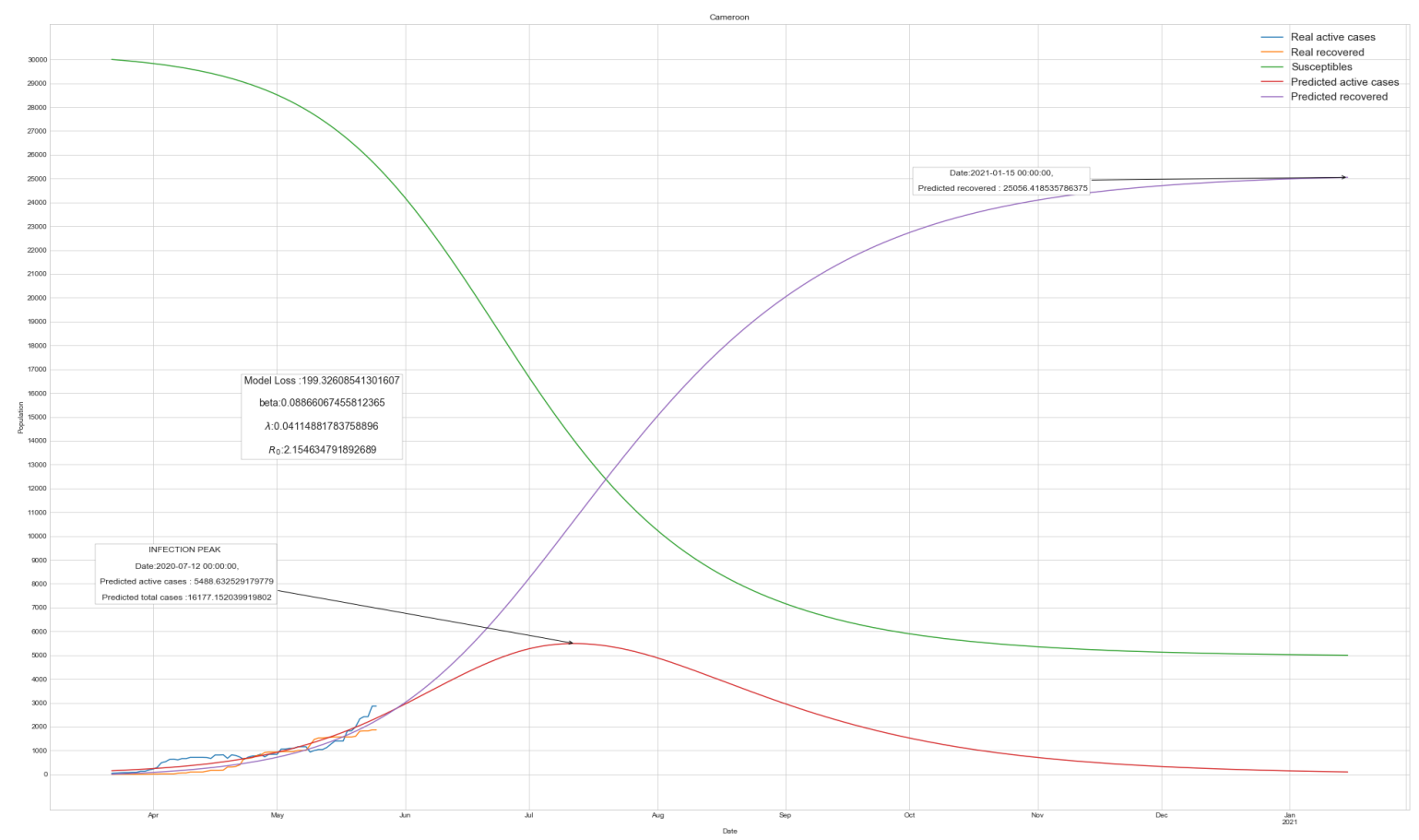

Figure 14. DETERMINISTIC SEIR $S_{0}=40000$

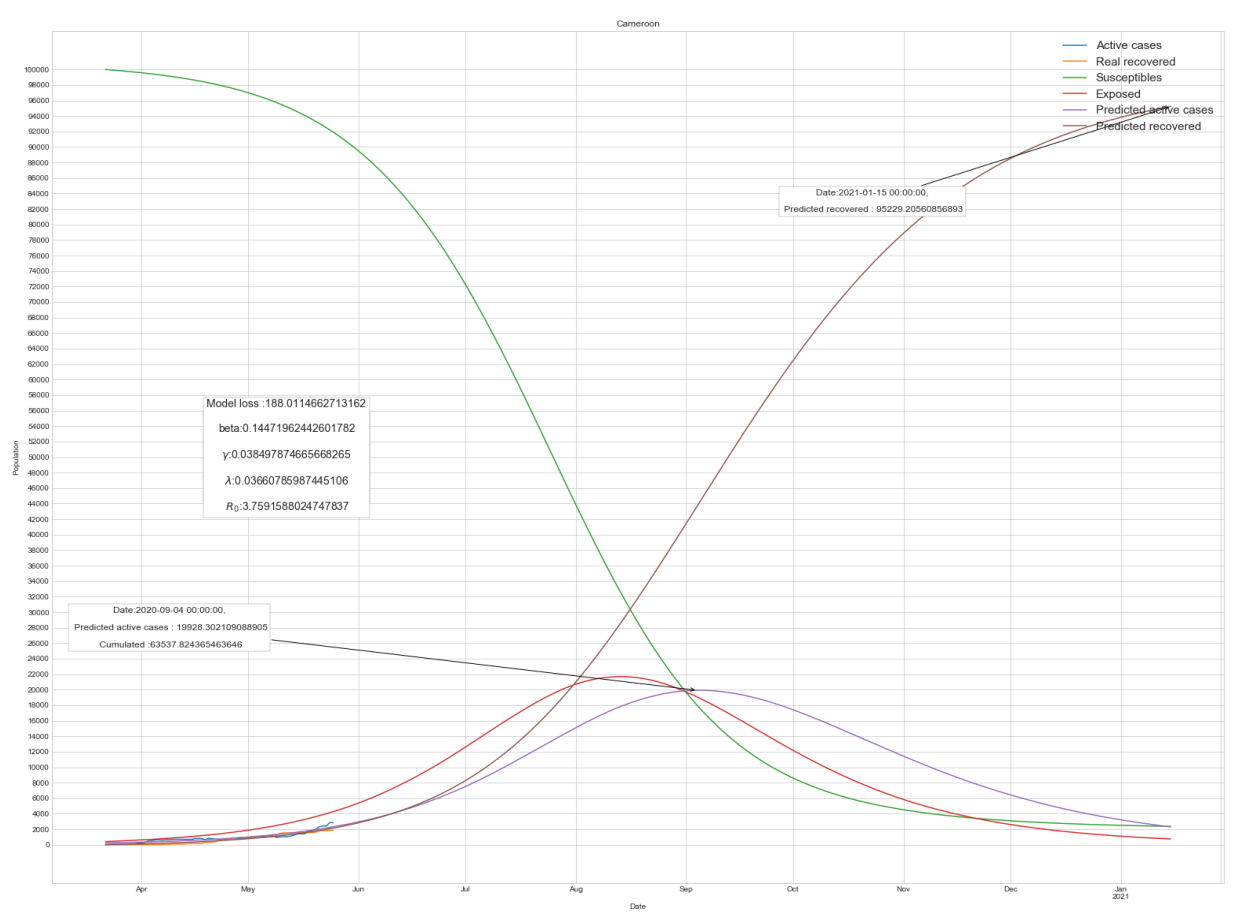

Figure 15. DETERMINISTIC SEIR $S_{0}=100000$ 
A summary of the main findings, limitations and implications of the model for policymakers is shown in Table 3

Table 3. DETERMINISTIC SEIR SUMMARY

\begin{tabular}{llllll}
\hline$S_{0}$ & $\begin{array}{l}\text { Infection peak } \\
\text { (Total cases) }\end{array}$ & Peak date & Model loss & $R_{0}$ & Parameters $(\beta, \gamma, \delta)$ \\
\hline 10000 & $2576[6952]$ & $22 / 06 / 2020$ & 206.00 & 6.44 & $(0.200,0.031,0.0344)$ \\
20000 & $4483[13359]$ & $18 / 07 / 2020$ & 195.60 & 4.210 & $(0.15268,0.036,0.034)$ \\
30000 & $6364.44[19653,73]$ & $01 / 08 / 2020$ & 191.97 & 4.14 & $(0.149,0.035,0.035)$ \\
40000 & $7798[21367]$ & $11 / 08 / 2020$ & 190.47 & 4.11 & $(0.146,0.035,0.0351$ \\
100000 & $19537[63224]$ & $09 / 09 / 2020$ & 188.268 & 3.84 & $(0.139,0.036,0.0356)$ \\
\hline
\end{tabular}

\subsection{Stochastic SEIR Model}

The stochastic SEIR is a novel extension of the existing SEIR model. It is denoted SSEIR. We present two versions of the SSEIR models. A model with homogeneous and heterogeneous noise is explained in details.

$$
\left\{\begin{array}{l}
\frac{d S(t)}{d t}=-\beta \frac{I(t)}{N} S(t)+B_{1}(t) \\
\frac{d E(t)}{d t}=\beta \frac{I(t)}{N} S(t)-\gamma E(t)+B_{2}(t) \\
\frac{d I(t)}{d t}=\gamma E(t)-\lambda I(t)+B_{3}(t) \\
\frac{d R(t)}{d t}=\lambda I(t)+B_{4}(t)
\end{array}\right.
$$

with $\left\{B_{1} \sim \mathcal{N}\left(0, \sigma_{1}\right), B_{2} \sim \mathcal{N}\left(0, \sigma_{2}\right), B_{3} \sim \mathcal{N}\left(0, \sigma_{3}\right), B_{4} \sim \mathcal{N}\left(0, \sigma_{4}\right)\right\}$.

The output of Stochastic SEIR with $S_{0}=10000,20000,40000$ and 100000 is presented in Figure 16, Figure 17, Figure 18 and Figure 19. 


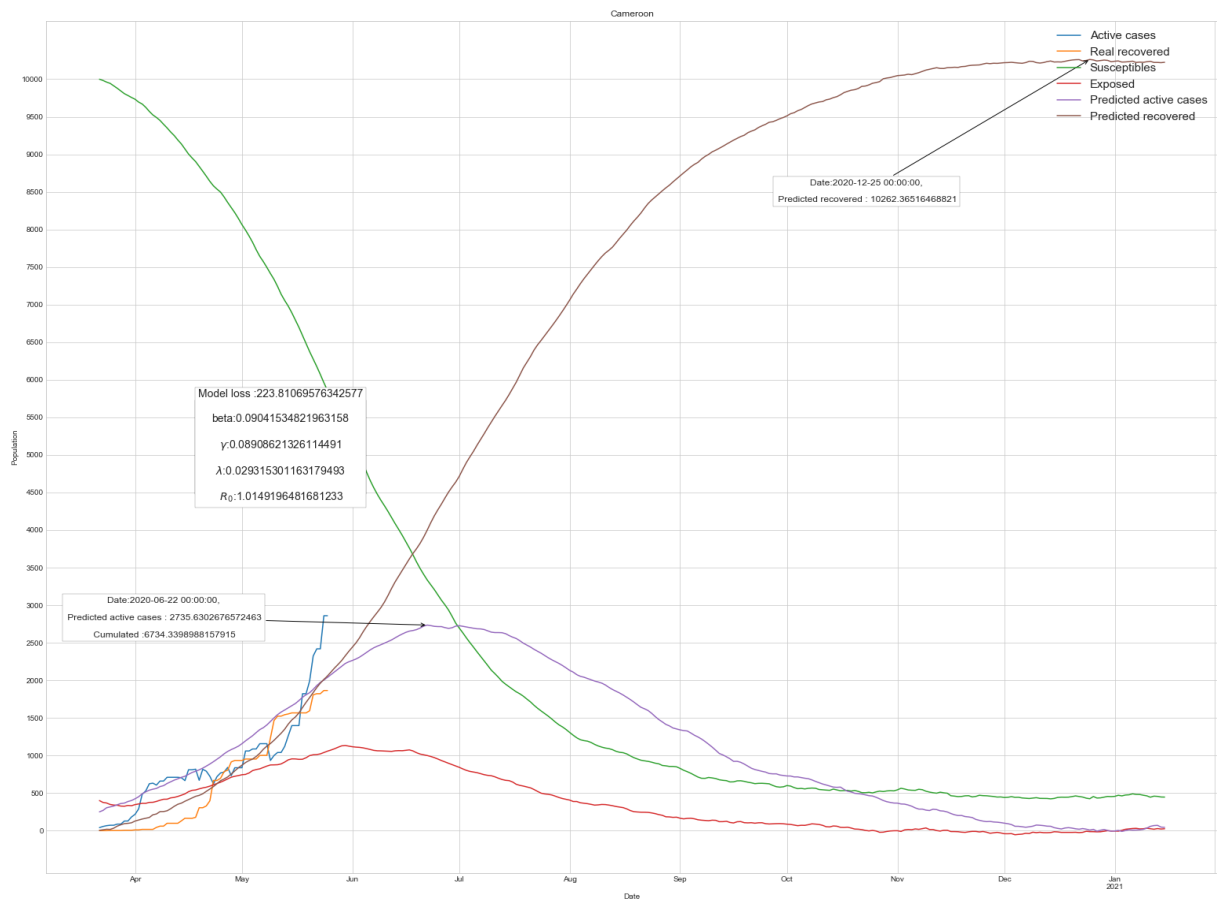

Figure 16. STOCHASTIC SEIR $S_{0}=10000$

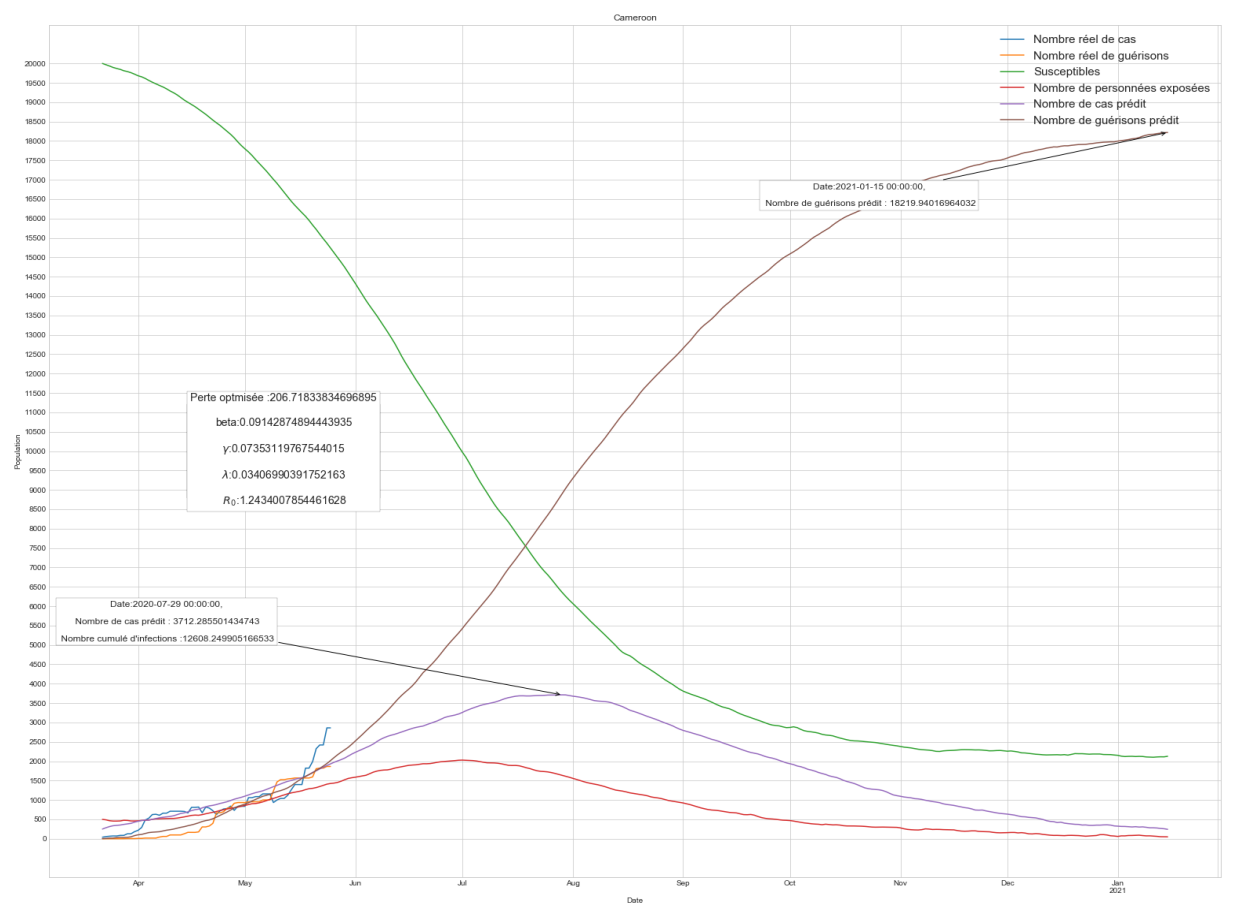

Figure 17. STOCHASTIC SEIR $S_{0}=20000$ 


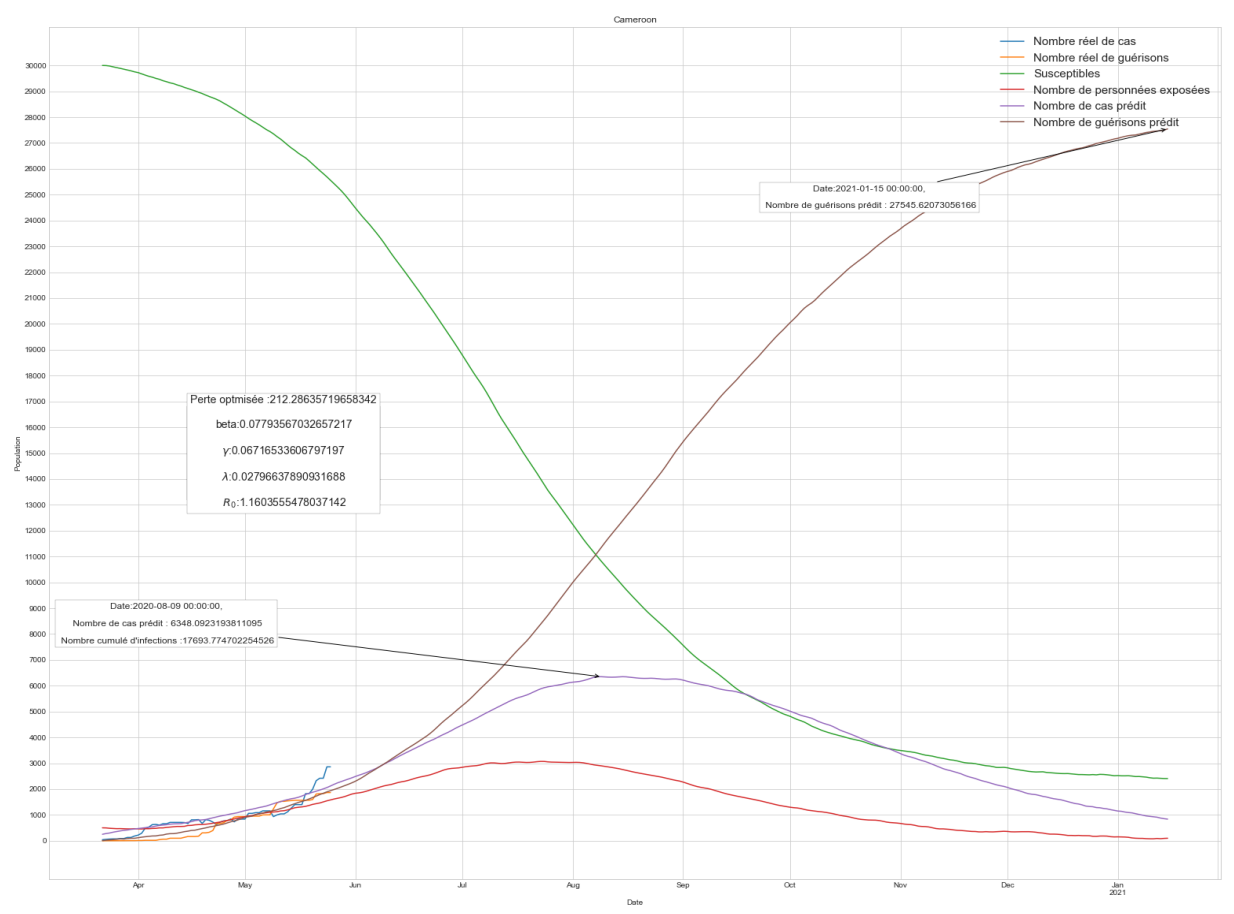

Figure 18. STOCHASTIC SEIR $S_{0}=30000$

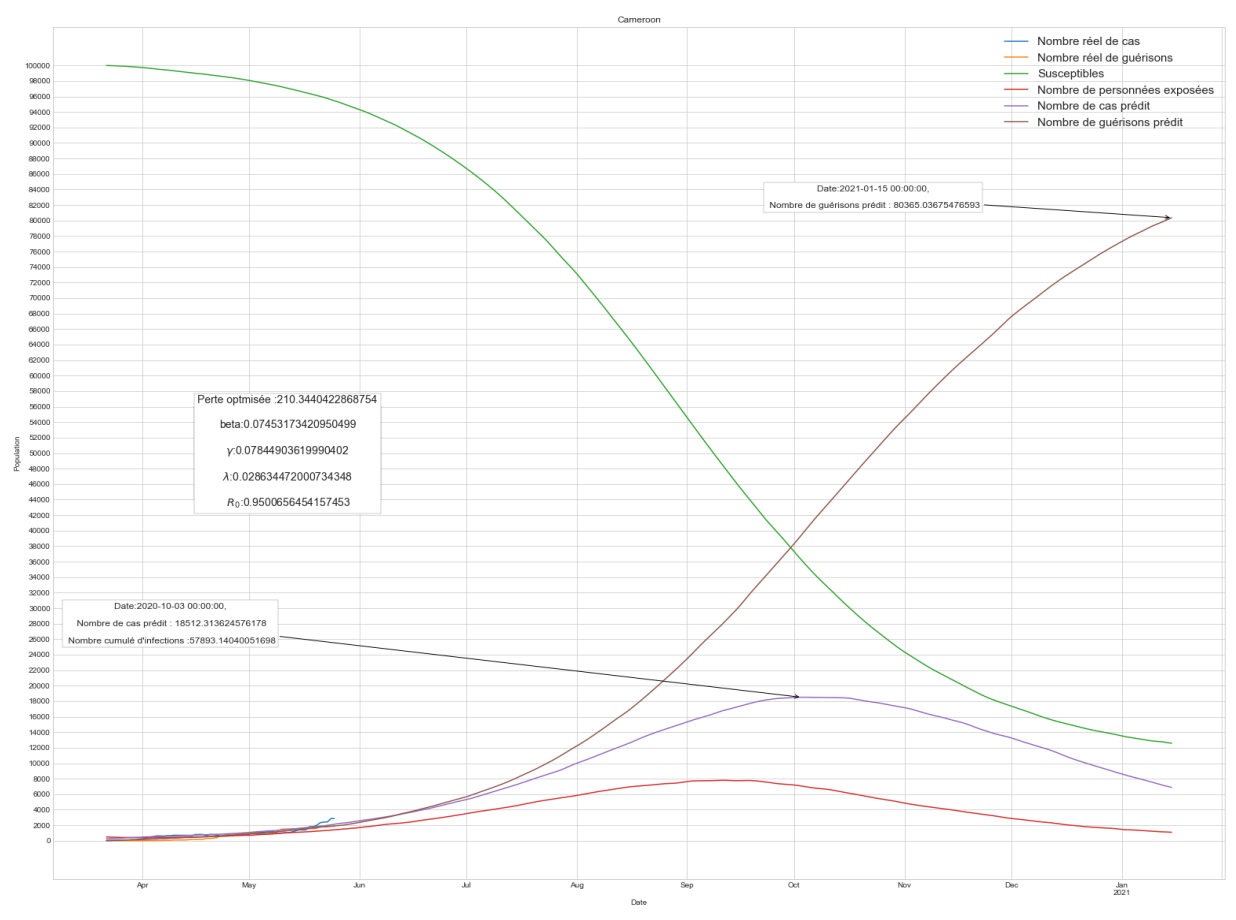

Figure 19. STOCHASTIC SEIR $S_{0}=10000$ 
The summary of the main findings, limitations and implications of the model with the data from Cameroon is shown in Table 4.

Table 4. STOCHASTIC SEIR SUMMARY

\begin{tabular}{|c|c|c|c|c|c|c|}
\hline$S_{0}$ & Noise & $\begin{array}{l}\text { Infection peak } \\
\text { [Total cases] }\end{array}$ & Peak date & $\begin{array}{l}\text { Model } \\
\text { loss }\end{array}$ & $R_{0}$ & $\begin{array}{l}\text { Parameters } \\
(\beta, \gamma, \lambda)\end{array}$ \\
\hline 10000 & $\begin{array}{l}\mathrm{N}(0,10), \mathrm{N}(0,10), \\
\mathrm{N}(0,10), \mathrm{N}(0,10)\end{array}$ & $2951[7521]$ & $22 / 06 / 2020$ & 198.28 & 1.143 & $(0.12,0.105,0.034)$ \\
\hline 20000 & $\begin{array}{l}\mathrm{N}(0,10), \mathrm{N}(0,10), \\
\mathrm{N}(0,10), \mathrm{N}(0,10)\end{array}$ & 3712 [12608] & $29 / 07 / 2020$ & 206.71 & 1.24 & $(0.091,0.07,0.034)$ \\
\hline 30000 & $\begin{array}{l}\mathrm{N}(0,10), \mathrm{N}(0,10), \\
\mathrm{N}(0,10), \mathrm{N}(0,10)\end{array}$ & 6348 [16953] & 09/08/2020 & 212.28 & 1.160 & $(0.077,0.067,0.027)$ \\
\hline 40000 & $\begin{array}{l}\mathrm{N}(0,10), \mathrm{N}(0,10), \\
\mathrm{N}(0,10), \mathrm{N}(0,10)\end{array}$ & 8497 [26614] & $25 / 08 / 2020$ & 210.173 & 1.29 & $(0.091,0.070,0.030)$ \\
\hline 100000 & $\begin{array}{l}\mathrm{N}(0,10), \mathrm{N}(0,10), \\
\mathrm{N}(0,10), \mathrm{N}(0,10)\end{array}$ & $18512[57893]$ & 03/10/2020 & 210.34 & 0.95 & $(0.074,0.0784,0.028)$ \\
\hline
\end{tabular}

\section{Parameter Optimization}

For the COVID-19 epidemic in Cameroon estimate the model parameters based on data from 22 March 2020 (day 1) to 25 May 2020 (day 61) and show how the progressive restrictions, including the most recent lockdown progressively enforced since 22 March 2020, have affected the spread of the epidemic. We also model possible longer-term scenarios illustrating the effects of noise on COVID-19 dynamic and also additionnal barrier measures such as social distancing and population-wide testing. The model parameters have been updated over time to reflect the progressive introduction of increased restrictions. In deterministic SIR $R_{0}$ was 2.44 in stochastic SIR $R_{0}$ was 2.32 , in determinstic SEIR $R_{0}$ was 4.15 , in stochastic SEIR $R_{0}$ was 1.30 . As we improve the model, the value of $R_{0}$ decreases as result of the introduction of basic social distancing, awareness of the epidemic, hygiene and behavioral recommendations, and early measures by the Cameroon government (for example, closing schools). Once the model parameters have been estimated on the basis of the available clinical data, the model enables us to reproduce and predict the dynamic evolution of the epidemic and to evaluate the possible underestimation or overestimation of the epidemic phenomenon based on current statistics, which are heavily subject to bias (for example, asymptomatic patients may get tested according to some protocols, not tested according to others). The model helps evaluate and predict the effect of the implementation of different guidelines and protocols (for example, more extensive screening for the disease or stricter social-distancing measures), which typically results in a change in the model parameters. The optimal values of the parameters can be found on the last column of each table.

\section{Algorithm}

We apply the The L-BFGS algorithm to find the optimal paramters of the model. The L-BFGS algorithm (Limited Mermory Broden-Fletcher-Goldfab-Shanno) is a very efficient algorithm for solving large scale problems. L-BFGS-B borrows ideas from the trust region methods while keeping the L-BFGS update of the Hessian and line search algorithms. Methods based completely on the trust region techniques exist and are freely available. The L-BFGS-B works for one iteration as follows :

1. Find the approximation of the Cauchy point for objective function as a quadratic form

2. Minimize the quadratic form

3. Perform a line search along new search direction

4. Update and check if the convergence is obtained 


\section{Stopping Criterion}

There are three different stopping criteria for the L-BFGS-B algorithm. First the program stops when the maximum number of iterations is reached. Or, the program stops when the decrease of the objective function becomes small enough. Or, the program stops when the norm of the projected gradient is small enough.

\section{Selection Criterion}

We will apply a criterion based on error bound to select the best model. The model with te smallest error bound is considered as the best model.

$$
\operatorname{Err}(N)=\frac{1}{N} \sqrt{\sum_{i}\left(X_{\text {realised }}(i)-X_{\text {predicted }}(i)\right)^{2}}
$$

\section{Stability}

The stability analysis in the present paper shows that despite the incorporation noise in the model, of the environmental variables, there is a similar pattern of stability exchange at $R_{0}=1$. Specifically, we establish the following results for the Covid 19 model: For $R_{0}<1$, there is a unique disease-free equilibrium which is both locally and globally asymptotically stable; this equilibrium becomes unstable when $R_{0}>1$. Meanwhile, there is a unique positive equilibrium which is locally asymptotically stable when $R_{0}>1$. A complete study of the epidemic global asymptotic stability for the full Covid 19 model is challenging, and we plan to pursue it in a separate paper.We will also briefly discuss the global asymptotic stability of the epidemic equilibrium for a simplified, linear case in the next section.

\subsection{Sensitivity Analysis with Respect to the Parameters $(\beta, \lambda, \gamma)$}

We now investigate the sensitivity of the model to parameter variations, focusing in particular on the parameters that can be influenced by policymakers: transmission parameters, related to lockdown measures $(\beta, \lambda, \gamma)$. To illustrate the effect of changing the parameter values in the model, our sensitivity analysis results are reported in Extended Table 5, 6, 7 and 8. Interestingly, the model is particularly sensitive to variations in the value of $\alpha$ and of $\varepsilon$. Increasing the parameters will increase the cumulative amount of infected individuals and the date affect the pic significantly.

We have also observed that if we increase the parameters of the model, from $+5 \%,+15 \%$ and $+20 \%$ we obtain a slight increase in the number of infected persons, the pic date but a decrease in $R_{0}$. Conversely, if we decrease the parameters of the model from $-5 \%,-15 \%$ and $-20 \%$, we also obtain a slight decrease in the number of infected persons, the pic of infection but an increase of $R_{0}$.

Table 5. DETERMINISTIC SIR parameters sensitivity

\begin{tabular}{lllllll}
\hline$S_{0}$ & P.s & $\begin{array}{l}\text { Infection Peak } \\
\text { [Total cases] }\end{array}$ & Peak date & Model Loss & $R_{0}$ & $\begin{array}{l}\text { Parameters } \\
(\beta, \gamma)\end{array}$ \\
\hline 30000 & $+10 \%$ & $5490[16221]$ & $02 / 07 / 2020$ & 199.32 & 2.15 & $(0.0975,0.045)$ \\
30000 & $-10 \%$ & $5486[16085]$ & $24 / 07 / 2020$ & 199.32 & 2.15 & $(0.079,0.037)$ \\
30000 & $-15 \%$ & $5486[16221]$ & $01 / 08 / 2020$ & 199.32 & 2.15 & $(0.075,0.0349)$ \\
30000 & $+15 \%$ & $5489[16073]$ & $27 / 06 / 2020$ & 199.32 & 2.15 & $(0.101,0.047)$ \\
30000 & $-20 \%$ & $5486[16176]$ & $09 / 08 / 2020$ & 199.32 & 2.15 & $(0.07,0.03)$ \\
30000 & $+20 \%$ & $5489[16082]$ & $23 / 06 / 2020$ & 199.32 & 2.15 & $(0.106,0.049)$ \\
\hline
\end{tabular}


Table 6. STOCHASTIC SIR parameters sensitivity

\begin{tabular}{llllllll}
\hline$S_{0}$ & P.s & Noise & $\begin{array}{l}\text { Infection Peak } \\
\text { [Total cases] }\end{array}$ & Peak date & M. Loss & $R_{0}$ & $\begin{array}{l}\text { Parameters } \\
(\beta, \gamma)\end{array}$ \\
\hline 30000 & $-10 \%$ & $\begin{array}{l}\text { N }(0,10), \mathrm{N}(0,10), \\
\mathrm{N}(0,10)\end{array}$ & $3178[11738]$ & $11 / 07 / 2020$ & 275.45 & 1.71 & $(0.089,0.052)$ \\
30000 & $+10 \%$ & $\begin{array}{l}\mathrm{N}(0,10), \mathrm{N}(0,10), \\
\mathrm{N}(0,10)\end{array}$ & $5332[15638]$ & $05 / 07 / 2020$ & 195.77 & 2.125 & $(0.090,0.042)$ \\
30000 & $+5 \%$ & $\begin{array}{l}\mathrm{N}(0,10), \mathrm{N}(0,10), \\
\mathrm{N}(0,10)\end{array}$ & $5841[17754]$ & $14 / 07 / 2020$ & 197.23 & 2.20 & $(0.090,0.040)$ \\
30000 & $-20 \%$ & $\begin{array}{l}\mathrm{N}(0,10), \mathrm{N}(0,10), \\
\mathrm{N}(0,10)\end{array}$ & $4816[16043]$ & $15 / 08 / 2020$ & 193.256 & 1.93 & $(0.064,0.033)$ \\
\hline
\end{tabular}

Table 7. DETERMINISTIC SEIR parameters sensitivity

\begin{tabular}{lllllll}
\hline$S_{0}$ & $\begin{array}{l}\text { Parameters } \\
\text { sensitivity }\end{array}$ & $\begin{array}{l}\text { Infection Peak } \\
{[\text { Total cases] }}\end{array}$ & Peak date & M. Loss & $R_{0}$ & $\begin{array}{l}\text { Parameters } \\
(\beta, \gamma, \lambda)\end{array}$ \\
\hline 30000 & $-5 \%$ & $6444.52[19639]$ & $07 / 08 / 2020$ & 192.176 & 2.95 & $(0.126,0.043,0.033)$ \\
30000 & $+5 \%$ & $6444[19674]$ & $25 / 07 / 2020$ & 192.176 & 2.95 & $(0.139,0.0472,0.037)$ \\
30000 & $-15 \%$ & $6049[18338]$ & $22 / 09 / 2020$ & 192.176 & 1.024 & $(0.073,0.072,0.0277)$ \\
30000 & $+15 \%$ & $6444.85[19640]$ & $14 / 07 / 2020$ & 192.176 & 1.677 & $(0.152,0.051,0.040)$ \\
30000 & $-20 \%$ & $6442[19661]$ & $02 / 09 / 2020$ & 192.176 & 2.948 & $(0.106,0.0358,0.02)$ \\
30000 & $+20 \%$ & $6444[19844]$ & $10 / 07 / 2020$ & 192.176 & 2.948 & $(0.159,0.054,0.042)$ \\
\hline
\end{tabular}

Table 8. STOCHASTIC SEIR parameters sensitivity

\begin{tabular}{|c|c|c|c|c|c|c|c|}
\hline$S_{0}$ & P.s & Noise & $\begin{array}{l}\text { Infection Peak } \\
\text { [Total cases] }\end{array}$ & Peak date & M. Loss & $R_{0}$ & $\begin{array}{l}\text { Parameters } \\
(\beta, \gamma, \lambda)\end{array}$ \\
\hline 30000 & $-5 \%$ & $\begin{array}{l}\mathrm{N}(0,10), \mathrm{N}(0,10), \\
\mathrm{N}(0,10), \mathrm{N}(0,10)\end{array}$ & $5892[18090]$ & $16 / 08 / 2020$ & 216.56 & 1.08 & $(0.083,0.076,0.030)$ \\
\hline 30000 & $+5 \%$ & $\begin{array}{l}\mathrm{N}(0,10), \mathrm{N}(0,10), \\
\mathrm{N}(0,10), \mathrm{N}(0,10)\end{array}$ & 5457 [16260] & $27 / 06 / 2020$ & 237.37 & 1.00 & $(0.414,0.025,0.053)$ \\
\hline 30000 & $-15 \%$ & $\begin{array}{l}\mathrm{N}(0,10), \mathrm{N}(0,10), \\
\mathrm{N}(0,10), \mathrm{N}(0,10)\end{array}$ & 5937 [18895] & 05/09/2020 & 201.008 & 1.07 & $(0.077,0.072,0.028)$ \\
\hline 30000 & $+15 \%$ & $\begin{array}{l}\mathrm{N}(0,10), \mathrm{N}(0,10) \\
\mathrm{N}(0,10), \mathrm{N}(0,10)\end{array}$ & $6492[19540]$ & $16 / 07 / 2020$ & 204.08 & 1.36 & $(0.111,0.082,0.037)$ \\
\hline 30000 & $-20 \%$ & $\begin{array}{l}\mathrm{N}(0,10), \mathrm{N}(0,10), \\
\mathrm{N}(0,10), \mathrm{N}(0,10)\end{array}$ & 3712 [12608] & $29 / 07 / 2020$ & 206.71 & 1.24 & $(0.091,0.07,0.034)$ \\
\hline 30000 & $+20 \%$ & $\begin{array}{l}\mathrm{N}(0,10), \mathrm{N}(0,10), \\
\mathrm{N}(0,10), \mathrm{N}(0,10)\end{array}$ & $6124[19627]$ & $16 / 07 / 2020$ & 192.95 & 1.2 & $(0.111,0.093,0.040)$ \\
\hline
\end{tabular}

- P.s: Parameters sensitvity

- M. Loss: Model loss

\subsection{Asymptotic behaviour of state variables (Stochastic SEIR Model)}

With fixed $S_{0}, E_{0}$ and defined $I_{0}$ as the initial number of infected patients which is known and $R_{0}=0$. Since $0 \leq S(t) \leq S(0) \leq N$ and $0 \leq R_{0} \leq R(t) \leq N$. This implies that $\lim _{t \rightarrow \infty} S(t)=S_{\infty}, \lim _{t \rightarrow \infty} R(t)=$ $R_{\infty}, \lim _{t \rightarrow \infty} E(t)=E_{\infty}$, therefore $I(\infty)=\lim _{t \rightarrow \infty} I(t)=N-S(\infty)-E(\infty)-R(\infty)$ exists. 


\section{Remarks}

1. The disease will die out when $I(\infty)=0, S_{\infty}=1-\epsilon,(\epsilon \in[0,1])$ and $R_{\infty}=\infty$

2. If $R_{0} \leq 1$, Then $I(t)$ decreases monitically to zero as $t \rightarrow \infty$.

3. If $R_{0}>1$, Then $I(t)$ starts increasing, reaches a maximumn then decreases to zero as $t \rightarrow \infty$. This caracterises an epidemic

\section{Results and Discussion}

Our findings provide researchers with a tool to assess the consequences of possible strategies, including lockdown and social distancing, as well as testing and contact tracing. Our simulation results, achieved by combining the model with the available data about the COVID-19 epidemic in Cameroon, suggest that enforcing strong social-distancing measures is urgent, necessary and effective, in line with other reports in the previous papers. The earlier the lockdown is enforced, the stronger the effect obtained. The model results also confirm the benefits of mass testing, whenever facilities are available. We believe these indications can be useful to manage the epidemic in Cameroon and other countries in Africa that are still in the early stages of outbreak. This article highlights the findings below:

1. The initial number of infected individuals is one of the key parameter for the COVID 19 propagation

2. By Adding noise to covid19 models we improve the results significantly.

3. The peak of the infected people strongly depends on the optimization of the parameters

4. The model selection relies only on the error bound of the model.

Assuming that the virus does not mutate and no vaccine is found, and we largely forgo testing and quarantining procedures, the number of deaths that would occur due to COVID-19 in Cameroon varies, and is improved as we improved the model. The peak value of active cases in a day and cumulative number of cases is calculated. The pandemic would affect a total of 15000 crore individuals by July.

There can be multiple reasons for this. Even though lock down has been imposed, it is not strictly followed. Violation of lock down means that temporary curbing of the pandemic does not occur. So the number of cases will continue to rise. Also testing and quarantining of the infected remains very poor in Cameroon The above results are what our deterministic an stochastic models predicts which are subject to the simplifying assumptions made while deriving them. Actual numbers are totally different in case stochasticity is important, which the present study ignores. Future deleterious mutations of COVID-19 may exacerbate the situation whereas development of an effective vaccine may mitigate the problem.

Practically, however, the strength and the success of the control measures would be limited by social and economic factors and available resources, and the combination of different prevention and intervention approaches would possibly achieve the best result. Other types of control measures, such as sanitation, sewage treatment, and water cleaning, can be also incorporated into the model and the resulting values of $R_{0}$ can be similarly calculated. Such information would provide useful guidelines for the public health administrations to effectively design disease control strategies and to properly scale their efforts.

\section{$5 \quad$ Limitations}

There are a few limitations in this study. As mentioned before, the model (1-5) is based on the assumption that $S_{0}$ is chosen large enough to solve the problem. This may not be true in the real world, especially when a long period of time is concerned. Next, the disease-induced mortality is not included in the model. the models do not account for migration; Datas were not collected by ourself; Model's parameters are limited ( 2 to 4 parameters); The computational time was short with determinstic models and longer with stochastic models; The current models are only suitable for countries with an acceptable number of infected people; Therefore, part of the results can be improved; Any factor that is not included in the models is considered as part of the noise; This research has been done under the pressure and the urgency of the current covid-19 situation. 


\section{Conclusion and Recommendations}

In this article we have provided a rough quantification of the error in estimating the actual number of infected people due to COVID-19 in Cameroon. Also, Our models can explain and predict the long-term effects of underdiagnosis, including the (apparently surprising) increased number of infections and fatalities, with sudden outbreaks after long silent periods. Our results demonstrate that restrictive social-distancing measures will need to be combined with widespread testing and contact tracing to end the ongoing COVID-19 pandemic. The model predictions in the long run remain sensitive to the initial conditions, but they are sensitive to the parameter values (and in particular extremely sensitive to some of these, as our sensitivity analysis has indicated), which are deeply uncertain and can vary due to several factors, such as population density, cultural habits, environmental conditions and age distribution of the population. The predictions must also consider parameter variations due to the measures imposed by the government. This is a fundamental aspect: in the long term, not imposing drastic measures leads to catastrophic outcomes, even when the initially affected population is a small fraction.(low). Social-distancing measures are modeled by reducing the infection coefficients $\beta, \lambda$ and $\gamma$. The infection peak time is not monotonic with increasing restrictions. Partial restrictions on population movements postpone the peak, while strong restrictions anticipate the peak. The modified version of mathematical model proposed and studied in this paper been proved more practical than the usual SIR model as it gives a better insight into covid 19 diseases and consequently enhances struggle against the spread of those diseases. It also revealed some remarkable result such as the effect of $S_{0}$ on the infection peak, the slight variation of parameters and the nature of the noise (homogenous vs heterogeneous). The modified version of the model can be used to study and describe more complex spread of disease

Data availability. We gathered epidemiological data from the following publicly available data sources: (https://raw.githubusercontent.com/CSSEGISandData/COVID-19/master/csse_covid_19_data/) and the Cameroon Ministry of Public Health (https://www.minsante.cm/).

All the epidemiological information we used is documented and can be provided upon request.

Code availabitity. The codes are available at:

https://drive.google.com/drive/folders/1Sph2fRxRzeEjMLuxEPDRv4Rs8KdPWSP4?usp=sharing

Acknowledgments. Professor Jimbo Henri Claver would like to thank the department of Mathematics of the Ecole Normale Superieure of Yaounde and Professors Andgiga and Tchantcho for the warm welcome and academic support during his stay as Visiting Professor at ENS. He equally thanks all members of the MMASFP(Multidisciplinary Modelling Actuary Finance Statistics and Probability) Research Group for the fruitful discussions in the weekly seminars. We would like to thank the anonymous referees for their valuable comments to improve this paper.

\section{References}

1. T. P. Velavan \& C. G. MeyerTe COVID-19 epidemic. Trop. Med. Int. Health25, 278-280 (2020).

2. Z. Wu, \& J. M. McGoogan, Characteristics of and important lessons from the coronavirus disease 2019 (COVID-19) outbreak in China: summary of a report of 72,314 cases from the Chinese center for disease control and prevention. JAMA 323, 1239-1242 (2020).

3. W.J. Guan,et al. Clinical characteristics of coronavirus disease 2019 in China.

4. N. Engl. J. Med. https://doi.org/10.1056/NEJMoa2002032 (2020).

5. WHO. Coronavirus Disease 2019 (COVID-19): Situation Report 76 (WHO, 2020).

6. A. Remuzzi, \& G.Remuzzi, COVID-19 and Italy: what next? Lancet Health.

7. Policy 395, 1225-1228 (2020).

8. A. Giufrida, \& P. Beaumont, Coronavirus: inquiry opens into hospitals atentre of Italy outbreak. Te Guardian (26 February 2020).

9. Ministero della Salute (Italian Ministry of Health). http://www.salute.gov.it/ imgs/C_17_notizie_4403_0_fle.pdf (5 April 2020). 
10. Y. Wang, Y. Chen, \& Q. Quin, Unique epidemiological and clinical features of the emerging 2019 novel coronavirus pneumonia (COVID-19) implicate special control measures. J. Med. Virol. 92, 568-576 (2020).

11. D. Fisman, C. Rivers, E. Lofgren \& M. S. Majumder, Estimation of MERS-Coronavirus reproductive number and case fatality rate for the Spring 2014 Saudi Arabia outbreak: insights from publicly available data. PLoS Curr https://doi.org/10.1371/currents.outbreaks.98d2f8f3382d84f390736cd5f5fe133c (2014).

12. S. Zhao, et al. Preliminary estimation of the basic reproduction number of novel coronavirus (2019-nCoV) in China, from 2019 to 2020: a data-driven analysis in the early phase of the outbreak. Int. J. Inf. Dis. 92, 214-217 (2020).

13. J. Read, J.R. Bridgen, D. A. T. Cummings, A. Ho, \& C. P. Jewell, Novel coronavirus 2019$\mathrm{nCoV}$ : early estimation of epidemiological parameters and epidemic predictions. Preprint at medRxiv https://doi.org/10.1101/2020.01.23.20018549 (2020).

14. H.C. Jimbo et al., Simulation and analysis of HIV-AIDS dynamics, International Journal of Healthcare and Computation vol 1, no 1, 2019.

15. O.Diekmann, \& J. A. P. Heesterbeek, Mathematical Epidemiology of Infectious Diseases: Model Building, Analysis and Interpretation (Wiley, 2000).

16. H. W. Hethcote, Te mathematics of infectious diseases. SIAM Rev. 42, 599-653 (2000).

17. F. Brauer, \& Castillo-Chavez, C. Mathematical Models in Population Biology and Epidemiology 2nd edn (Springer, 2012).

18. W. O. Kermack, \& A. G. McKendrick, A contribution to the mathematical theory of epidemics. Proc. R. Soc. Lond. 115, 700-721 (1927).

19. Q. Lin, et al. A conceptual model for the coronavirus disease 2019 (COVID-19) outbreak in Wuhan, China with individual reaction and governmental action. Int. J. Inf. Dis. 93, 211-216 (2020).

20. C. Anastassopoulou, L.Russo, A. Tsakris, \& C. Siettos, Data-based analysis, modelling and forecasting of the COVID-19 outbreak. PLoS One 15, e0230405 (2020).

21. F. Casella, Can the COVID-19 epidemic be managed on the basis of daily data? Preprint at https://arxiv.org/abs/2003.06967 (2020)

22. G.M.Nakamura, A.S. Martinez . Hamiltonian dynamics of the SIS epidemic model with stochastic fluctuations. Sci Rep. 2019;9(1):1-9. doi:10.1038/s41598-019-52351-x

23. B. Li, Periodic orbits of autonomous ordinary differential equations: Theory and applications, Nonlinear Analysis, 5 (1981), 931-958.

24. G. Li and J. Zhen, Global stability of an SEI epidemic model with general contact rate, Chaos Solitons Fractals, 23 (2005), 997-1004.

25. M. Y. Li, J. R. Graef, L. Wang and J. Karsai, Global dynamics of a SEIR model with varying total population size, Mathematical Biosciences, 160 (1999), 191-213.

26. M. Y. Li and J. S. Muldowney, Global stability for the SEIR model in epidemiology, Mathematical Biosciences, 125 (1995), 155-164

27. H.C, Jimbo et al., Co-Evolutionary Algorithm for Analyzing Gene Expression Data, Advance in Computer Science Research, Editor A. Dadvaud, Atlantis Press, Pp 120 - 126, 2016

28. H. C., Jimbo et al., Modelling Cancer Chemotherapy with Side-Effects, IASTED, Acta Press, 838-010, 2016 Canada

29. T. Yamada, H. C. Jimbo, S. Ishii, M. Nishiyama, K. Hong and Y. Sakumura, Identification of a Molecular System that Regulates Growth Cone Membrane Potential during growth cone Guidance, BMC NEUROSCIENCE, 201112 (Suppl 1) P28.

30. H.C. Jimbo et al., A Dynamical Model of Cancer Chemotherapy with Disturbance, GECCO Campagnon 12, ACM, New York, USA, GECCO12, Pp1417- 1418, 